\section{IDDF2019-ABS-0225 LNCRNA W01880 IS DOWNREGULATED IN GASTRIC CANCER CELL AND INHIBITED PROLIFERATION AND METASTASIS}

Haojun Song*, Bujiang Wang, Xiaoyun Ding. Department of Gastroenterology, Ningbo First Hospital, China

\subsection{6/gutjnl-2019-IDDFabstracts.42}

Background Gastric cancer (GC) is one of the common cancers in China, and its pathogenesis is still unknown. Recent studies have reported that long non-coding RNAs (lncRNAs) are important regulators of GC. However, the role and mechanism of lncRNAs in GC are poorly understood. In this study, we characterized a novel lncRNA termed w01880 in GC tissues and cell lines.

Methods We used reverse transcription-quantitative polymerase chain reaction (RT-qPCR) to assayed w01880 levels in GC tissues and cell lines. Lentiviral vector was used to manipulate the expression of w01880 (IDDF2019-ABS-0225 Figure 1C). Biological functions of w01880 were analyzed using Cell Counting Kit-8 (CCK-8) and Wound Healing in vitro.

Results We analyzed a cohort of 64 patients and found that w01880 was significantly down-expressed in GC tissues compared with paired adjacent normal tissues ((figure 1A), $P<0.001)$. Combined with clinicopathological features, the expression level of w01880 in GC patients was correlated with depth of invasion, lymph node metastasis and TNM staging ((table 1), $P<0.05)$. Then, we found that w01880 is almost low expression in GC cell lines ((figure $1 \mathrm{~B}), P<0.05$ ). The in vitro overexpression of w01880 inhibited the proliferation and Invasion of GC cell (MNK-45) ((figure 1D,E), $P<0.001)$.
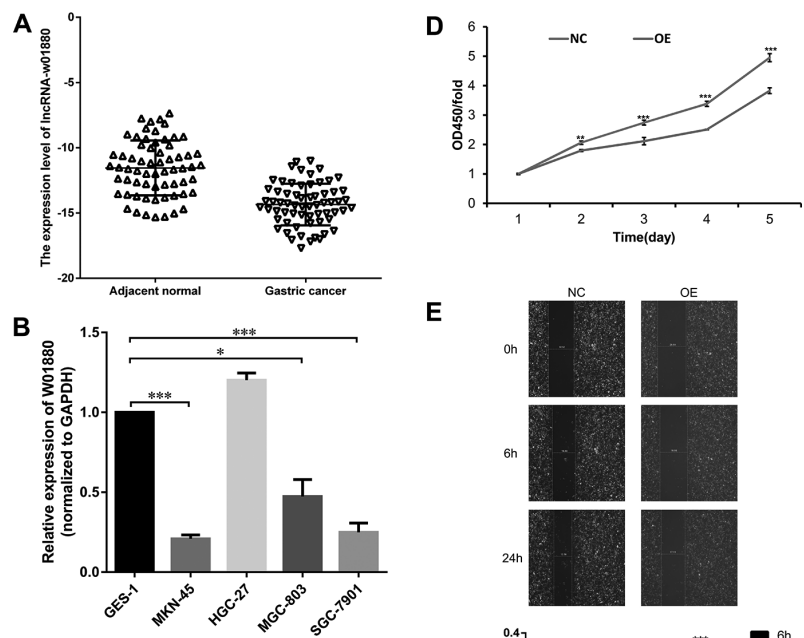

C
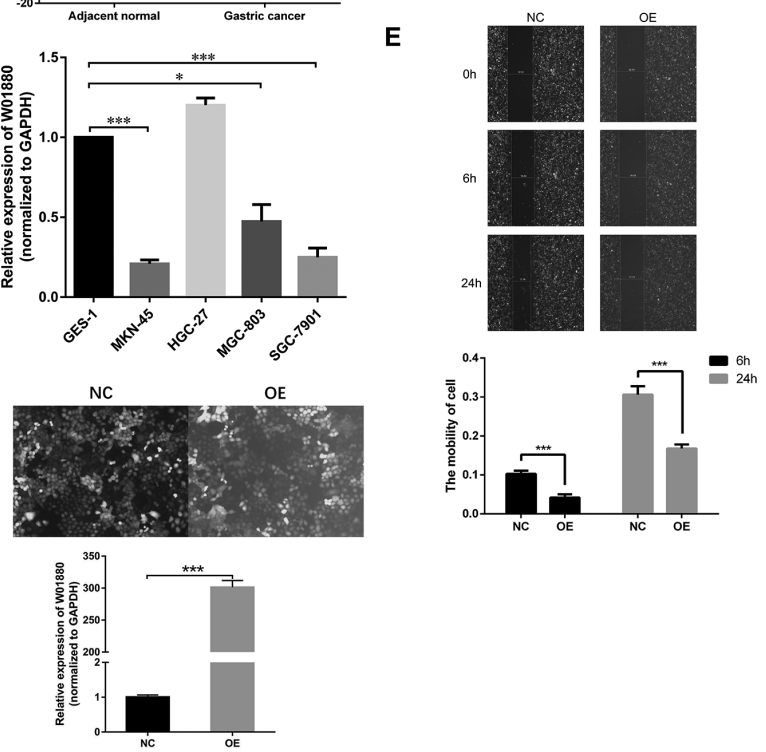

Abstract IDDF2019-ABS-0225 Figure 1
Abstract IDDF2019-ABS-0225 Table 1 Correlation between w01880 expression and clinic pathologic characteristics

\begin{tabular}{llll}
\hline Characteristics & $\begin{array}{l}\text { Number of } \\
\text { patients }\end{array}$ & $\begin{array}{l}\text { w01880 } \\
\text { Mean } \pm \text { SD }(-\Delta \mathrm{Ct})\end{array}$ & P value \\
\hline$T$ stage & & & $<0.05$ \\
T1\&T2 & 22 & $-13.32 \pm 0.95$ & \\
T3\&T4 & 42 & $-14.85 \pm 1.66$ & $<0.05$ \\
Lymph node metastasis & & & \\
N0 & 21 & $-13.04 \pm 1.23$ & $<0.05$ \\
N1\&N2\&N3 & 43 & $-14.95 \pm 1.41$ & \\
TNM stage & & & \\
I\&II & 30 & $-13.08 \pm 1.05$ & \\
III\&IV & 34 & $-14.41 \pm 1.19$ & \\
\hline
\end{tabular}

Conclusions Our data suggested that w01880 functioned as a tumor suppressor IncRNA in GC, which provided a potential biomarker and therapeutic target for GC.

\section{IDDF2019-ABS-0227 MANIPULATION OF GUT MICROBIOTA IN VITRO MODEL OF COLORECTAL CANCER: STRONG ADHERENCE ABILITY OF LACTOBACILLUS RHAMNOSUS}

Norfilza Mokhtar*, Konken Wong, Raja Affendi Raja Ali. GUT research group, Faculty of Medicine, Universiti Kebangsaan Malaysia, Malaysia; Department of Microbiology, Universiti Kebangsaan Malaysia, Malaysia

\subsection{6/gutjnl-2019-IDDFabstracts.43}

Background Intestinal flora is closely linked with the occurrence of colorectal cancer (CRC). The role of probiotic to target this disease is a promising strategy beyond its role as basic nutrition and maintaining homeostasis. Lactobacillus is a type of lactic acid bacteria. Lactobacillus rhamnosus is one of the strain that has the ability to survive in low $\mathrm{pH}$ and bile, able to colonise and adhere to intestinal epithelial layer, that makes it a suitable candidate as probiotic. Meanwhile, Fusobacterium nucleatum ATCC 25586 is a gram-negative bacteria, was documented to be enriched in CRC. The aim of this study was to determine the ability of Lactobacillus rhamnosus ATCC 53103 to displace and compete with Fusobacterium nucleatum ATCC 25586.

Methods We used 2 CRC cell lines (SW480 and HCT116) as the host cells. Three different assays i.e. protection assay, displacement assay and competitive assay were performed and repeated three times in total. Multiplicity of infection (MOI) of 100:1 (bacteria:cells) was used and plated on blood agar 4 hours post-infection of cells. Colony-forming unit (cfu) was used to indicate the number of adhered bacteria.

Results Lactobacillus rhamnosus showed higher binding capacity on host cells than Fusobacterium nucleatum with ratio 94:1 and 215:1 respectively based on the competitive assay. In addition, Lactobacillus rhamnosus could lower the adherence ability of Fusobacterium nucleatum up to $80 \%$ in the protection and competitive assays, however only $20 \%$ in the displacement assay.

Conclusions This study highlighted the possible option of probiotic Lactobacillus rhamnosus as a preventive measure to stop the proliferative effects of CRC cells. The strong adherence of Lactobacillus rhamnosus could potentially reduce the invasion of the Fusobacterium nucleatum involved in carcinogenesis of colorectal cancer. 